\title{
Modification of the Fluctuation Dynamics of Ultrathin Wetting Films
}

\author{
C. Clavaud $\odot,{ }^{1}$ M. Maza-Cuello, ${ }^{1}$ C. Frétigny, ${ }^{1}$ L. Talini $\odot,{ }^{2, *}$ and T. Bickel ${ }^{3}$ \\ ${ }^{1}$ CNRS, Sciences et Ingénierie de la Matière Molle, ESPCI Paris, PSL Research University, \\ Sorbonne Université, 75005 Paris, France \\ ${ }^{2}$ CNRS, Surface du Verre et Interfaces, Saint-Gobain, 93300 Aubervilliers, France \\ ${ }^{3}$ Univ. Bordeaux, CNRS, Laboratoire Ondes et Matière d'Aquitaine (UMR 5798), F-33400 Talence, France
}

(Received 19 February 2021; accepted 13 May 2021; published 4 June 2021)

\begin{abstract}
We report on the effect of intermolecular forces on the fluctuations of supported liquid films. Using an optically induced thermal gradient, we form nanometer-thin films of wetting liquids on glass substrates, where van der Waals forces are balanced by thermocapillary forces. We show that the fluctuation dynamics of the film interface is strongly modified by intermolecular forces at lower frequencies. Data spanning three frequency decades are in excellent agreement with theoretical predictions accounting for van der Waals forces. Our results emphasize the relevance of intermolecular forces on thermal fluctuations when fluids are confined at the nanoscale.
\end{abstract}

DOI: 10.1103/PhysRevLett.126.228004

When shared with air or another fluid, a liquid interface appears macroscopically flat but is actually corrugated by thermal motion at smaller scales. Although of subnanometric amplitude, thermal capillary waves play a central role in phenomena such as drop formation [1], film breakup [2], jet destabilization $[3,4]$, as well as wetting $[5,6]$ or dewetting [7] of liquid films on solid substrates. As first described by Vrij [8] and Sheludko [9], the rupture of a nondraining liquid film originates from the amplification of interfacial fluctuations with wavelengths larger than a critical value, which is set by the balance between surface tension and intermolecular forces. The lifetime of metastable supported thin liquid films is therefore ruled by the thermal roughness of their interface. In turn, fluctuations are themselves modified as the thickness of the liquid layer decreases since confinement enhances hydrodynamic dissipation [10,11].

Intermolecular forces are also expected to have a significant effect on the fluctuations of ultrathin films. Indeed, dipolar interactions between the solid substrate, the liquid layer, and the gas phase become relevant when the thickness of the film is smaller than a few tens of nanometers [12]. The resulting van der Waals forces can be either attractive or repulsive. In the case of attractive interactions, the fluctuations are amplified by intermolecular forces and the film is predicted to be unstable [13-16]. This is confirmed by experimental observations on

Published by the American Physical Society under the terms of the Creative Commons Attribution 4.0 International license. Further distribution of this work must maintain attribution to the author(s) and the published article's title, journal citation, and DOI. dewetting polymer films of nanometric thickness [7]. Still, in this nonequilibrium situation, measurements are limited to the growth of the more unstable mode and the full spectrum of thermal capillary waves remains unexplored. In contrast, the fluctuation amplitude of a liquid layer with repulsive liquid-air and liquid-solid interactions is expected to decrease under the action of intermolecular forces. This qualitatively explains the formation and stability of ultrathin films of wetting liquids, such as the precursor films involved in the spreading of a macroscopic droplet. Nevertheless, the effect of van der Waals forces on interfacial fluctuation has never been evidenced experimentally. More generally, investigations regarding thermal capillary waves at the interface of nanoscale liquid films remain almost exclusively theoretical.

Thermal fluctuations of liquid interfaces were first characterized more than forty years ago using light scattering techniques [17]. They proved to be an accurate and noninvasive probe of the macroscopic properties of liquids, e.g., surface tension or viscoelasticity, that can be deduced from the capillary wave spectrum for an infinite medium [18]. Owing to experimental limitations, measurements have long been limited to very thick liquid layers. The effect of confinement has been addressed more recently thanks to the development of novel techniques based on either the deflection of a reflected laser beam $[19,20]$ or $\mathrm{x}$-ray scattering [21,22]. Both techniques differ by the range of the wave vectors that can be probed in the experiments. Small wavelength x-ray measurements are better suited for highly viscous fluids such as high molecular weight polymer melts, whereas laser deflection can be used for simple, low-viscosity liquids. Enhancement of experimental accuracy now makes it possible to investigate the modification of the fluctuation dynamics in very 
thin films $[22,23]$. Hydrodynamic boundary conditions at the liquid-solid interface were also successfully tested [24].

However, the contribution of intermolecular forces on the capillary spectrum has never been evidenced yet. The experimental challenges are multiple since stable films of controlled nanometric thickness must be produced in the first place. Then, interfacial deformations have to be probed at the molecular scales and over long times. To achieve these goals, we make use of the thermocapillary effect to create ultrathin films of wetting liquids. We then measure the fluctuation dynamics of the interface in a large frequency range using the previously developed surface fluctuation specular reflection technique [19]. We further compare the experimental data with theoretical predictions based on the lubrication approximation. Our analysis therefore shows that the thickness of the liquid layer results from a balance between thermocapillary and intermolecular forces, and that the fluctuation spectra are dominated by van der Waals forces at low frequencies.

Silicone oils (Rhodorsil) of two different viscosities (9.3 and $19 \mathrm{mPas}$ at room temperature) are spin coated on colored glass substrates (Hoya Longpass optical filters, diameter $50 \mathrm{~mm}$, thickness $2.5 \mathrm{~mm}$ ) to form films of uniform initial thickness, between 250 and $1150 \mathrm{~nm}$. The orange color of the glass substrate (optical index $n=1.526$ ) is chosen for its poor but nonzero absorption of the He-Ne laser light (wavelength $632.8 \mathrm{~nm}$ ). The spincoated films are placed in the setup described in Fig. 1. The laser is strongly focused on the film with a $\times 50$ microscope objective. The other optical elements are used to avoid back laser light and to collect the light reflected by the interface

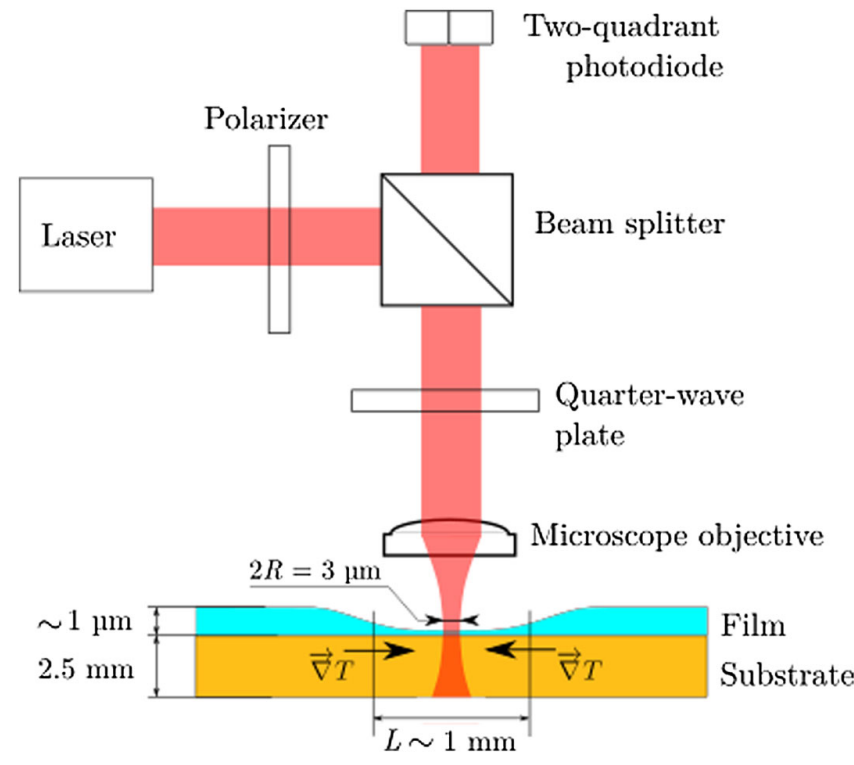

FIG. 1. Schematized experimental setup (not to scale). The laser is focused on the liquid film spread on a colored glass substrate. Light absorption by the substrate creates a thermal gradient in the liquid layer over the length scale $L$, which also defines the extension of the film thinning. on a two-quadrant photodiode. Special care is taken to suppress parasitic light on the photodiode and to place the liquid film at the laser beam waist within the Rayleigh length $(\approx 20 \mu \mathrm{m})$. The reflected beam is centered on the two quadrants and the variations of its position are detected by computing the power spectrum density (PSD) of the difference between the voltages delivered by the quadrants. Owing to either low- or high-frequency parasitic noise, fluctuation spectra are obtained for frequencies ranging from $1 \mathrm{~Hz}$ to $1 \mathrm{kHz}$.

As previously described [24], the laser is used for three different purposes. First, it induces a thermocapillary flow in the liquid film, so that the thickness decreases locally. Second, the laser reflections at the air-liquid and liquidsolid interface interfere and are collected on the photodiode, allowing accurate measurements of the film thickness by interferometry. Lastly, the laser is used to measure the fluctuations of the slope of the liquid layer interface, which result in fluctuations of the position of the reflected beam.

We first focus on the thinning of the oil film due to the thermocapillary effect. The colored glass substrate is slightly heated by the laser beam. The temperature of the film therefore increases by thermal diffusion within a few seconds. Convection is negligible in the liquid phase since the thermal Péclet number is much smaller than unity. The resulting surface tension gradient creates a liquid flow directed away from the laser beam, which induces a local thinning of the film (see Fig. 1). Since the absorption length of the glass substrate is very large compared to its thickness $(=2.5 \mathrm{~mm})$, it is the latter millimetric length that sets the lateral scale of the thermal gradient, and hence of film thinning. The extension of the thin flat region is therefore 3 orders of magnitude larger than the actual beam size $(R=1.5 \mu \mathrm{m})$.

To describe the thinning of the film, we denote $\theta(r)=$ $T(r)-T_{0}$ the temperature rise, with $T_{0}$ the room temperature and $r$ the radial coordinate with origin at the beam center. The temperature rise being small $(<1 \mathrm{~K}$, see below), we neglect the variations of the physical properties of the liquid that are at most $1 \%$ of their equilibrium values. The temperature dependance of the surface tension is only accounted for in the thermocapillary stress $(\approx 0.1 \mathrm{~Pa})$ resulting from the thermal gradient $\left(\approx 10^{3} \mathrm{~K} \mathrm{~m}^{-1}\right)$. The film thickness $h(r, t)$ then follows the thin-film equation $[25,26]$

$\partial_{t} h=\frac{1}{3 \eta r} \partial_{r} r h^{3}\left\{\partial_{r}\left[-\gamma_{0} \nabla^{2} h+\phi^{\prime}(h)\right]+\frac{3 \gamma_{\theta}}{2 h} \frac{d \theta}{d r}\right\}$,

with $\eta$ the liquid viscosity, $\gamma_{0}$ the equilibrium surface tension, and $\gamma_{\theta}=|\partial \gamma / \partial \theta|$ the derivative of surface tension with respect to temperature. Since we focus on submicron scales, gravity can safely be neglected. The first term in parentheses corresponds to the Laplace pressure. The second term $\phi^{\prime}(h)$ is the derivative of the van der Waals interaction potential 
(per unit area); it is the opposite of the so-called disjoining pressure. Consistently with the literature on oil films [27], we assume $\phi^{\prime}(h)=A /\left(6 \pi h^{3}\right)$ with a negative Hamaker constant $A<0$. Both intermolecular and Laplace forces oppose the thinning of the film, which is driven by the thermocapillary stress - the third term in parentheses.

A comprehensive study of the film thinning kinetics will be detailed in a future work. We focus here on the stationary version of Eq. (1). Neglecting the Laplace pressure term, a double integration of Eq. (1) yields the stationary thickness under the beam $h_{0}=h(r=0)$ [26]

$$
h_{0}=\left(\frac{|A|}{6 \pi \gamma_{\theta} \theta(0)}\right)^{1 / 2},
$$

where $\theta(0)$ is the maximum excess temperature. The thickness is therefore set by $\theta(0)$ but is independent of both the initial film thickness, the viscosity and the details of the temperature profile. With $\gamma_{\theta}=6.5 \times 10^{-5} \mathrm{Nm}^{-1} \mathrm{~K}^{-1}$ [28] and taking for instance $\theta(0)=1 \mathrm{~K}$, we predict a stationary thickness $h_{0} \approx 9 \mathrm{~nm}$ for $|A|=10^{-19} \mathrm{~J}$.

We show in Fig. 2 an example of the measured variation of an oil film thickness. After an initial decrease, the stationary state is reached within a few minutes. The thickness value at the plateau $h_{0}=10.0 \pm 0.3 \mathrm{~nm}$ remains remarkably stable for several hours. Let us emphasize that the liquid plateau is very flat since it actually extends over the millimetric size of the thermal gradient. The original Eq. (2) expresses the balance between intermolecular and thermocapillary forces. From this perspective, our experiment is a novel analogous to the thin film pressure balance, in which the disjoining pressure of a liquid film is measured

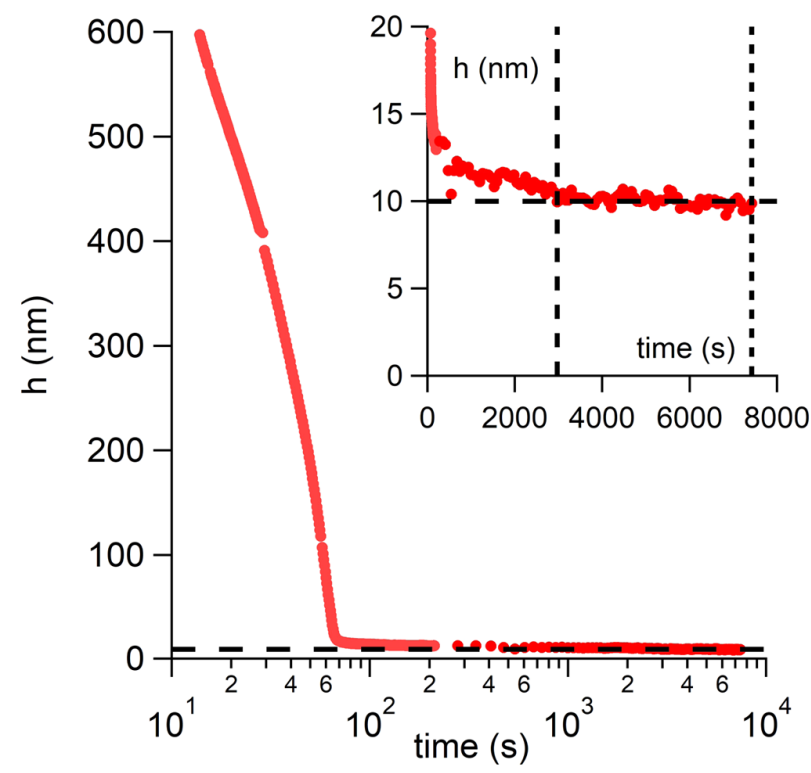

FIG. 2. Thickness of film of a silicone oil (viscosity $9.3 \mathrm{mPa} \mathrm{s}$ ) under the laser beam as a function of time. Inset shows details of the plateau corresponding to $h_{0}=10.0 \pm 0.3 \mathrm{~nm}$. at equilibrium with an imposed pressure [29]. Here, the film thickness is simply controlled by adjusting the intensity of the laser beam. Using a variable neutral density, we can check that thickness varies as predicted with laser intensity (see Fig. S1 in the Supplemental Material [26]). Stationary thicknesses ranging from 8 to $30 \mathrm{~nm}$ are obtained by tuning the laser intensity.

In the following, we take advantage of the long-lasting stability of the thin films to measure fluctuation spectra over a wide frequency range. We especially focus on low frequencies that are most sensitive to intermolecular forces. As already mentioned, the same laser is used to measure interfacial fluctuations. More precisely, it is the fluctuating slope of the liquid surface that results in fluctuations in the position of the specularly reflected beam off the surface. In contrast to scattering experiments, in which a single surface mode is selected, the contributions of all the surface modes are measured but with different weights. Actually, the finite radius $R$ of the beam sets the length scale of the measurement and the main contribution to the signal comes from the fluctuating modes with wave vectors $q \sim 1 / R$ [19]. The power spectral density (PSD) can then be quantitatively expressed as $S(\omega) \propto \int_{0}^{\infty} P(q, \omega) \Phi(q) d q$-see Ref. [24] for a comprehensive discussion of $S(\omega)$ and all the parameters on which it depends. The weight function $\Phi(q)$ only depends on the beam characteristics (size and divergence), and is maximum for $q=2 \pi / R$. The mode density $P(q, \omega)$ represents the PSD of the liquid film: $P(q, \omega)=\left\langle\left|\tilde{h}_{\mathbf{q}, \omega}\right|^{2}\right\rangle$, with $\tilde{h}_{\mathbf{q}, \omega}$ the Fourier transform of the height $h(\mathbf{r}, t)$.

The mode density can be obtained in the lubrication regime $q h_{0} \ll 1$, with $h_{0}$ a few tens of nanometers. Note that the lubrication theory cannot always be used to describe thin film dynamics; for instance, it has been shown recently to fail to describe the very last moments before film rupture [30]. Here however, the weight function $\Phi(q)$ is maximum for $q \sim 1 / R$, with $R=1.5 \mu \mathrm{m} \gg h_{0}$. The lubrication approximation is therefore fully legitimate (see Supplemental Material for the derivation of the fluctuation spectra beyond the lubrication approximation [26]). Our derivation of the stochastic version of the thin film Eq. (1) then follows Ref. [13]. Assuming small deviations with respect to the stationary height $h_{0}$, we can write $h(\mathbf{r}, t)=h_{0}+\delta h(\mathbf{r}, t)$ with $|\delta h| \ll h_{0}$. The thin film equation can then be linearized and one obtains at lowest order [26]

$$
\partial_{t} \delta h=\frac{h_{0}^{3}}{3 \eta}\left[\phi^{\prime \prime}\left(h_{0}\right) \nabla^{2} \delta h-\gamma_{0} \nabla^{4} \delta h\right]+\kappa \boldsymbol{\nabla} \cdot \mathbf{f},
$$

where the stochastic term $\mathbf{f}=\left(f_{x}, f_{y}\right)$ follows a Gaussian distribution with ensemble average $\left\langle f_{i}(\mathbf{r}, t)\right\rangle=0$ and $\left\langle f_{i}(\mathbf{r}, t) f_{j}\left(\mathbf{r}^{\prime}, t^{\prime}\right)\right\rangle=\delta_{i j} \delta\left(\mathbf{r}-\mathbf{r}^{\prime}\right) \delta\left(t-t^{\prime}\right)$. The noise amplitude is $\kappa=\sqrt{2 k_{B} T_{0} h_{0}^{3} /(3 \eta)}$. It is important to note that the 
thermocapillary stress happens to be irrelevant as far as fluctuations are concerned. Indeed, although it sets the stationary thickness of the film, its contribution to the fluctuation spectrum is several orders of magnitude smaller than the capillary stress and the disjoining pressure [26]. It is then straightforward to show that the mode density $P(q, \omega)$ is a Lorentzian function of the frequency

$$
P(q, \omega)=\frac{k_{B} T_{0}}{\pi} \frac{\Gamma\left(q, h_{0}\right)}{\omega^{2}+\omega_{q}^{2}} .
$$

The relaxation rate is given by $\omega_{q}=\left(\gamma_{0} / \lambda^{2}\right) \Gamma\left(q, h_{0}\right) \times$ $\left(1+q^{2} \lambda^{2}\right)$, with $\Gamma\left(q, h_{0}\right)=q^{2} h_{0}^{3} /(3 \eta)$ the dissipation kernel. Since gravity is negligible, the capillary length is replaced by $\lambda=\sqrt{2 \pi h_{0}^{4} \gamma_{0} /|A|}$. This length scale lies in the micrometer range for typical values $|A| \approx 10^{-19} \mathrm{~J}$, $\gamma_{0} \approx 10^{-2} \mathrm{~N} \mathrm{~m}^{-1}$, and $h_{0} \approx 10^{-8} \mathrm{~m}$. As a consequence, the contributions from both capillary and intermolecular forces are equally significant in the PSD. Interestingly, $\lambda$ also corresponds to the critical wavelength of the unstable modes leading to the rupture of metastable films $[8,13]$. Equation (4) moreover reveals that the main contribution of van der Waals forces is to shift the low cutoff frequency of the Lorentzian function. Indeed, no modification of the capillary spectrum is expected at high frequencies, where $P(q, \omega) \sim \omega^{-2}$. In contrast, one expects a signature of van der Waals forces on the fluctuation spectrum at low frequencies, below a few tens of $\mathrm{Hz}$ taking the same numerical values as above.

We thus anticipate a significant contribution of intermolecular forces for thicknesses smaller than a few tens of nanometers and at low frequencies. Figure 3 displays the experimental spectra of two different oil films with two different thicknesses: 12.9 (viscosity 9.3) and $30.3 \mathrm{~nm}$ (viscosity $19 \mathrm{mPa}$ s). The data are obtained in the stationary regime and averaged over at least 20 spectra (the acquisition time of each spectrum being $50 \mathrm{~s}$ ). The experimental spectra are in excellent agreement with the theoretical prediction obtained by numerical integration of the mode density Eq. (4). Tabulated values of viscosity, surface tension, and refractive indexes were used, together with measured values of film height and beam radius. The only adjusted parameter is therefore the Hamaker constant: we get $|A|=1.2 \times 10^{-19}$ and $|A|=2 \times 10^{-19} \mathrm{~J}$ for the less and more viscous oil, respectively. For comparison, the theoretical spectra without the contribution of intermolecular forces are also shown. Clearly, it is the low-frequency part of spectra that is most strongly modified by van der Waals forces. One can notice that the Hamaker constants for silicon oils on glass are 1 order of magnitude larger than for alkane films on mica, but they are very close to alkane films on steel [27]. Silicone oils being known to be particularly difficult to remove from solid surfaces, this

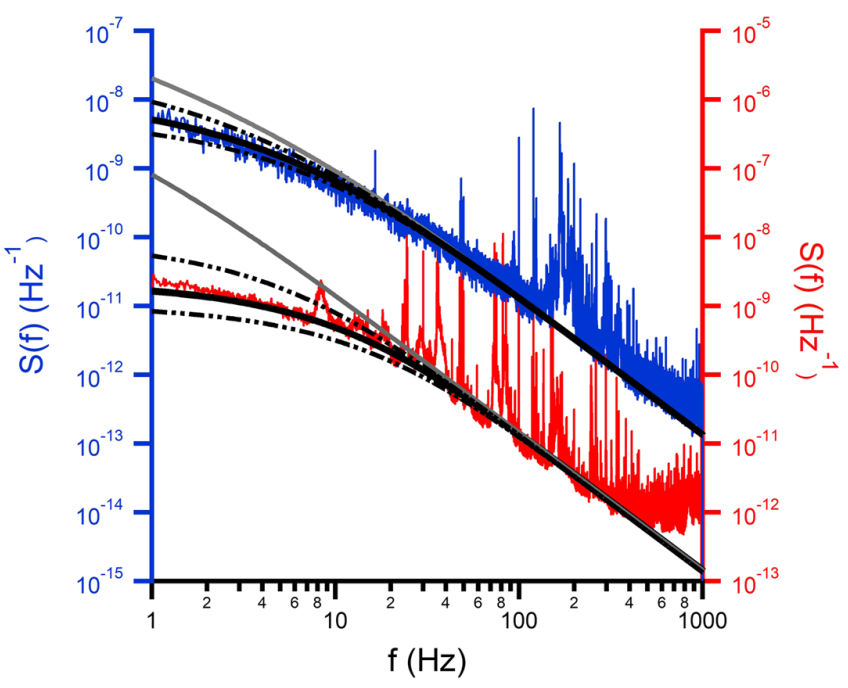

FIG. 3. Fluctuation spectra of oil films of thickness 30.3 (viscosity $19 \mathrm{mPas}$, blue, left axis) and $12.9 \mathrm{~nm}$ (viscosity $9.3 \mathrm{mPa} \mathrm{s}$, red, right axis). Spurious peaks result from parasitic noise either of mechanical or electromagnetical origin. For each spectrum, the full lines represent the theoretical spectra without (gray lines) and with (black lines) intermolecular forces effects. Dash-dotted lines correspond to spectra computed with Hamaker constants differing by $\pm 50 \%$.

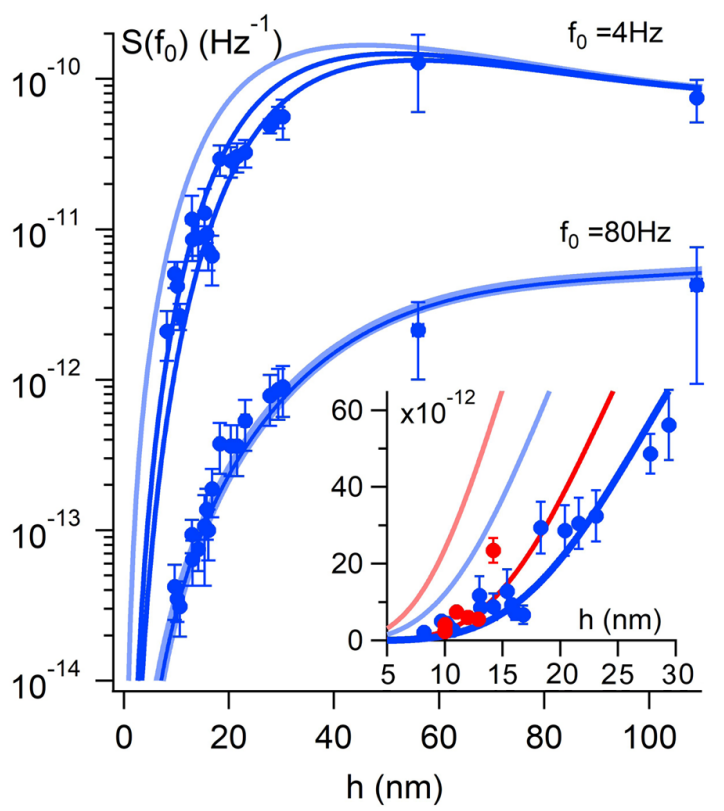

FIG. 4. Fluctuation spectra (in logarithmic scale) of oil films (viscosity $19 \mathrm{mPas}$ ) at 4 and $80 \mathrm{~Hz}$, as a function of film thickness. The full lines correspond to theoretical predictions without (lighter lines) and with (darker lines) intermolecular forces, with $|A|=10^{-19}$ and $|A|=2 \times 10^{-19} \mathrm{~J}$. Inset: Fluctuation spectra (in linear scale) at $4 \mathrm{~Hz}$ of the two oil films with viscosity 19 (blue circles, same data as main figure) and $9.3 \mathrm{mPa}$ (red circles). Vertical error bars correspond to the statistical uncertainty. Horizontal error bars are too small to appear. 
finding consistently indicates a strong repulsion between glass-oil and oil-air surfaces.

Additional experiments are performed where the film thickness is varied by adjusting the laser intensity. In each experiment, the fluctuation spectra of a stationary thin film are measured at two different frequencies, respectively, 4 and $80 \mathrm{~Hz}$. The corresponding values of the PSD, respectively averaged over a 0.8 and $16 \mathrm{~Hz}$-wide frequency range, are reported in Fig. 4 as a function of the film thickness. Note that for the thicker films, measurements are conducted before reaching the plateau as the film is thinning down. Only three spectra could then be recorded and the large error bars result from poor averaging. All data points are in excellent agreement with our theoretical predictions. Figure 4 confirms that the effect of intermolecular forces is most significant for the lower frequencies and for smaller thicknesses. In contrast, intermolecular forces do not modify the spectra at the larger frequencies, even for film thicknesses below $10 \mathrm{~nm}$. Similar data are obtained for the two different oils. Note that the Hamaker constants are expected to be very close since optical indexes of the two silicon oils only differ by $0.1 \%$.

To summarize, we have developed a novel method to form oil films of tunable nanometric thicknesses using a laser-induced thermocapillary effect. The films are stable for several hours, allowing for the characterization of the fluctuation dynamics of their interface. We thus present the first evidence of the contribution of intermolecular forces on the fluctuation spectra of supported liquid films, over a large frequency range. The experimental data are fully described by our theoretical predictions accounting for van der Waals forces, the Hamaker constant being the only adjusted parameter. A significant decrease of the amplitude of the spectra is observed at low frequencies, showing the relevance of intermolecular forces in nanometric liquid layers. Our results therefore provide new insight on the dynamics of ultrathin liquid films.

This project has received funding from the European Union's Horizon 2020 research and innovation program under the Marie Sklodowska-Curie Grant Agreement No. 754387.

*laurence.talini@espci.fr

[1] Y. Hennequin, D. G. A. L. Aarts, J. H. van der Wiel, G. Wegdam, J. Eggers, H. N.W. Lekkerkerker, and D. Bonn, Drop Formation by Thermal Fluctuations at an Ultralow Surface Tension, Phys. Rev. Lett. 97, 244502 (2006).

[2] E. Chatzigiannakis and J. Vermant, Breakup of Thin Liquid Films: From Stochastic to Deterministic, Phys. Rev. Lett. 125, 158001 (2020).

[3] M. Moseler and U. Landman, Formation, stability, and breakup of nanojets, Science 289, 1165 (2000).
[4] J. Petit, D. Rivière, H. Kellay, and J.-P. Delville, Break-up dynamics of fluctuating liquid threads, Proc. Natl. Acad. Sci. U.S.A. 109, 18327 (2012).

[5] B. Davidovitch, E. Moro, and H. A. Stone, Spreading of Viscous Fluid Drops on a Solid Substrate Assisted by Thermal Fluctuations, Phys. Rev. Lett. 95, 244505 (2005).

[6] J. C. Fernandez-Toledano, T. D. Blake, and J. De Coninck, Contact-line fluctuations and dynamic wetting, J. Colloid Interface Sci. 540, 322 (2019).

[7] R. Fetzer, M. Rauscher, R. Seemann, K. Jacobs, and K. Mecke, Thermal Noise Influences Fluid Flow in Thin Films during Spinodal Dewetting, Phys. Rev. Lett. 99, 114503 (2007).

[8] A. Vrij, Possible mechanism for the spontaneous rupture of thin, free liquid films, Discuss. Faraday Soc. 42, 23 (1966).

[9] A. Sheludko, Thin liquid films, Adv. Colloid Interface Sci. 1, 391 (1967).

[10] J. Jäckle, The spectrum of surface waves on viscoelastic liquids of arbitrary depth, J. Phys. Condens. Matter 10, 7121 (1998).

[11] M. L. Henle and A. J. Levine, Capillary wave dynamics on supported viscoelastic films: Single and double layers, Phys. Rev. E 75, 021604 (2007).

[12] T. Bickel, Probing nanoscale deformations of a fluctuating interface, Europhys. Lett. 106, 16004 (2014).

[13] K. Mecke and M. Rauscher, On thermal fluctuations in thin film flow, J. Phys. Condens. Matter 17, S3515 (2005).

[14] L. G. MacDowell, J. Benet, and N. A. Katcho, Capillary Fluctuations and Film-Height-Dependent Surface Tension of an Adsorbed Liquid Film, Phys. Rev. Lett. 111, 047802 (2013).

[15] Y. Zhang, J. E. Sprittles, and D. A. Lockerby, Molecular simulation of thin liquid films: Thermal fluctuations and instability, Phys. Rev. E 100, 023108 (2019).

[16] Y. Zhang, J. E. Sprittles, and D. A. Lockerby, Thermal capillary wave growth and surface roughening of nanoscale liquid films, J. Fluid Mech. 915, A135 (2021).

[17] M. A. Bouchiat and J. Meunier, Spectre des fluctuations thermiques de la surface libre d'un liquide simple, J. Phys. II (France) 32, 561 (1971).

[18] D. Langevin, Light Scattering by Liquid Surfaces and Complementary Techniques (Marcel Dekker, New York, 1992).

[19] A. Tay, C. Thibierge, D. Fournier, C. Fretigny, F. Lequeux, C. Monteux, J. P. Roger, and L. Talini, Probing thermal waves on the free surface of various media: Surface fluctuation specular reflection spectroscopy, Rev. Sci. Instrum. 79, 103107 (2008).

[20] T. Mitsui and K. Aoki, Measurements of liquid surface fluctuations at sub-shot-noise levels with Michelson interferometry, Phys. Rev. E 87, 042403 (2013).

[21] X. Hu, Z. Jiang, S. Narayanan, X. Jiao, A. R. Sandy, S. K. Sinha, L. B. Lurio, and J. Lal, Observation of a lowviscosity interface between immiscible polymer layers, Phys. Rev. E 74, 010602(R) (2006).

[22] K. J. Alvine, Y. Dai, H. W. Ro, S. Narayanan, A. R. Sandy, C. L. Soles, and O. G. Shpyrko, Capillary Wave Dynamics of Thin Polymer Films over Submerged Nanostructures, Phys. Rev. Lett. 109, 207801 (2012). 
[23] B. Pottier, E. Verneuil, L. Talini, and O. Pierre-Louis, Surface fluctuations of liquids confined on flat and patterned solid substrates, Phys. Rev. E 89, 052403 (2014).

[24] B. Pottier, C. Frétigny, and L. Talini, Boundary Condition in Liquid Thin Films Revealed Through the Thermal Fluctuations of Their Free Surfaces, Phys. Rev. Lett. 114, 227801 (2015).

[25] A. Oron, S. H. Davis, and S. G. Bankoff, Long-scale evolution of thin liquid films, Rev. Mod. Phys. 69, 931 (1997).

[26] See Supplemental Material at http://link.aps.org/ supplemental/10.1103/PhysRevLett.126.228004 for the experimental variations of stationary thickness with the laser intensity. It also includes a complete description of the model and additional information regarding the relevant physical processes, as well as a comprehensive study of the fluctuation dynamics beyond the lubrication approximation.

[27] N. V. Churaev, Surface forces in wetting films, Colloid J. 65, 263 (2003).

[28] E. Ricci, R. Sangiorgi, and A. Passerone, Density and surface tension of dioctylphthalate, silicone oil and their solutions, Surf. Coat. Technol. 28, 215 (1986).

[29] B. Saramago, Thin liquid wetting films, Curr. Opin. Colloid Interface Sci. 15, 330 (2010).

[30] D. Moreno-Boza, A. Martínez-Calvo, and A. Sevilla, Stokes theory of thin-film rupture, Phys. Rev. Fluids 5, 014002 (2020). 